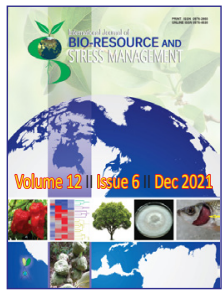

\title{
Soil Nutrient Analysis Underneath Grewia optiva Population
}

\author{
H. P. Sankhyan ${ }^{1}$, Jyoti Dhiman ${ }^{1}, K^{\prime}$ ishan Chand ${ }^{2}$, Prachi ${ }^{1}$ Karishma ${ }^{1}$ and Bhupender Negi ${ }^{1}$
}

${ }^{1}$ Dept. of Tree Improvement and Genetic Resources, ${ }^{2}$ Dept. of Siliviculture and Agroforestry, College of Forestry, Dr. Y.S Parmar University of Horticulture and Forestry, Nauni-Solan, Himachal Pradesh (173 230), India

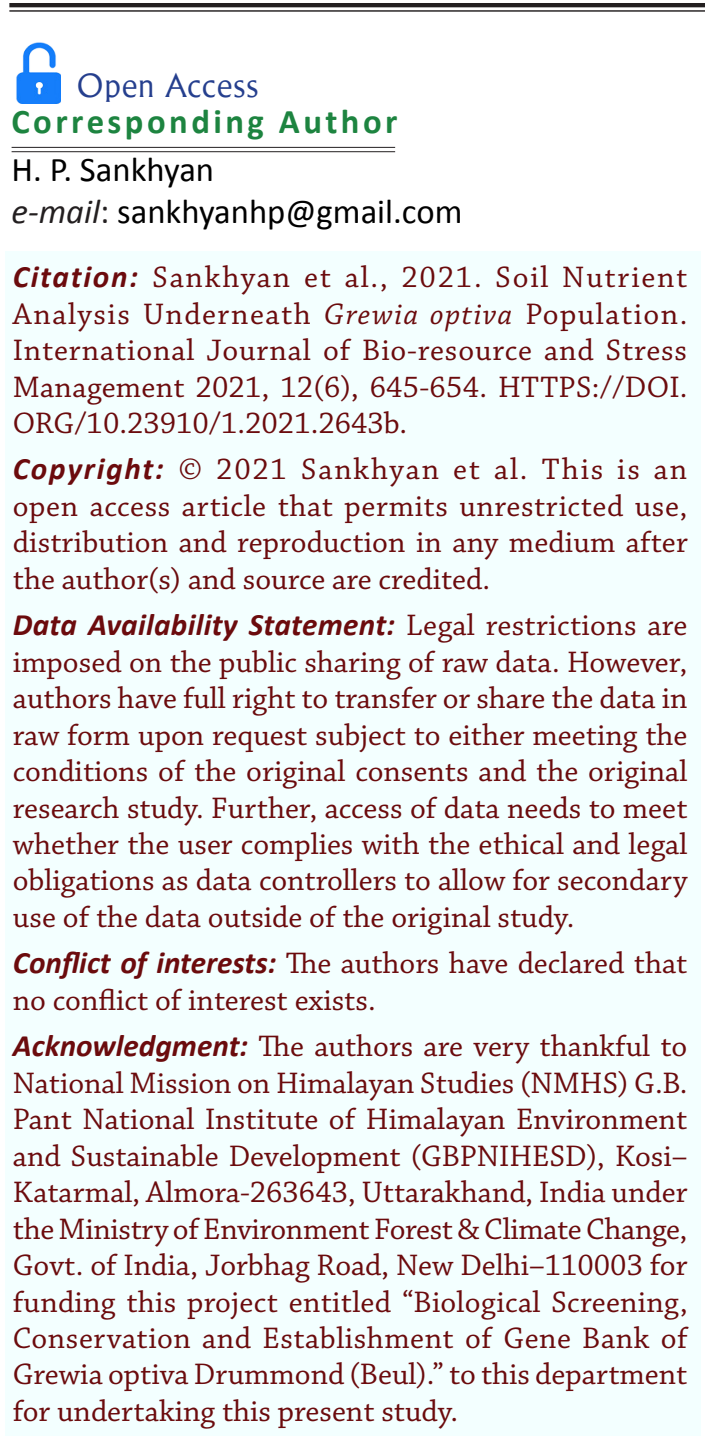

\begin{abstract}
The present study was conducted during May and November, 2019 and 2020 and here we quantify the impact of soil nutrient variation on the population growth of Grewia optiva Drummond of different districts with variable climate and heterogeneous soils. The impacts on growth parameters (tree height, crown spread, leaf traits, fruit dimensions) of selected populations of Grewia optiva Drummond statistically analyzed using ANOVA and Pearson correlation coefficient. Five composite soil samples were taken at 15-30 cm depth, on seven selected districts of Himachal Pradesh viz., total 35 samples were analyzed for physiochemical properties. Soil of Mandi district registered with acidic $\mathrm{pH}$ as compared to soil $\mathrm{pH}$ of other districts. There was significant difference in $\mathrm{pH}$, $\mathrm{OC}, \mathrm{EC}, \mathrm{N}, \mathrm{P}, \mathrm{K}$ and in Bulk density within selected population of each district. $\mathrm{pH}$ range of moderately acidic to moderately alkaline (according to standard soil classification) founded best for growth of this species. There was highly positive correlation observed between Nitrogen and leaf area (0.33), Phosphorus and leaf area (0.45). 100 leaf fresh weight showed highly positive correlation with Nitrogen (0.37) and organic carbon (0.39). 100 leaf dry weight showed highly positive correlation with Nitrogen and Organic Carbon. Crown spread showed highly positive correlation with Organic carbon (0.29), Nitrogen (0.38) and with Phosphorus (0.30). Moderately Positive correlation observed between Potassium and 100 fruit dry weight (0.15). $50 \%$ of soil texture observed as sandy loamy and sandy clay loamy.
\end{abstract}

Keywords: Fodder, Grewia optiva, growth, macronutrient, plant community, soil

\section{Introduction}

Grewia optiva, commonly known as Biul/Bihul/Bhimal, belongs to family Tiliaceae. This species is preferred by hilly farmers of the Uttarakhand, Himachal Pradesh, Nepal etc., for its attributes like palatability, faster growth, ease of propagation and forage yield (Mukherjee et al., 2018). It provides fodder during lean season, when no alternate to green fodder is available. (Katoch et al., 2017). It has over 70 percent potential DM digestibility and effective degradability (56.7 Per cent) which makes it superior energy source for ruminants (Singh et al., 1989). The genus Grewia consists of some 150 species in world, out of which 42 species are found in Indian subcontinent (Bhagta et al., 2021).

Soil consists of definite chemical, physical, mineralogical and biological properties, which provides a medium for plant growth (Thakre et al.,

Article History

RECEIVED on $29^{\text {th }}$ September 2021 RECEIVED in revised form on $10^{\text {th }}$ December 2021 ACCEPTED in final form on $28^{\text {th }}$ December 2021 
2012). The knowledge of physiochemical properties viz; organic carbon, available Nitrogen $(\mathrm{N})$, Phosphorus $\left(\mathrm{P}_{2} \mathrm{O}_{5}\right)$, Potassium $\left(\mathrm{K}_{2} \mathrm{O}\right), \mathrm{pH}$, electrical conductivity, soil texture and bulk density of soil is also important to determine the available nutrient status in soil and to develop specific fertilizer recommendations. (Sumithra et al., 2013). As pH is a good indicator of the balance of available nutrients in the soil whereas, Electrical Conductivity can almost be viewed as the quantity of available nutrients in the soil. (Smith and Doran, 1996). The response of trees to increasing atmospheric $\mathrm{CO}_{2}$ concentrations is often mediated by the availability of nutrients in the soil (Schleppi et al., 2019). Whether terrestrial ecosystems, forests, crop land trees are sources or sinks for $\mathrm{CO}_{2}$ and their growth will ultimately depend on interactions of the $\mathrm{C}$ cycle with the cycles of nutrients, especially nitrogen (N) and phosphorus (P) (Ellsworth et al., 2017). An increased production of exoenzymes has been found in several studies with $\mathrm{CO}_{2}$ enrichment, and this effect has depended on the availability of $\mathrm{N}$ in the soil (Drake et al., 2013, Meier et al., 2017, Ochoa-Hueso et al., 2017). Nitrogen (N) is one of the most important biological elements for plants, agricultural crops and forest trees, because it is a component of amino acids, proteins, genetic materials, pigments, and other key organic molecules (Chen et al., 2014, Ji et al., 2015, Liu et al., 2018). N has an irreplaceable role in organ construction, material metabolism, fruit yield, and the quality formation of fruit trees (Bai et al., 2016). Soil nitrogen (N) deficiencies can affect the photosynthetic N-use efficiency (PNUE), mesophyll conductance $\left(g_{\mathrm{m}}\right)$, and leaf $\mathrm{N}$ allocation (Tang et al., 2019). Potassium (K) is used for flowering purpose, it is also required for building of protein, photosynthesis, fruit quality and reduction of diseases (Valente et al., 2012). Potassium is an activator of dozens of important enzymes, such as protein synthesis, sugar transport, $\mathrm{N}$ and $\mathrm{C}$ metabolism, and photosynthesis. It plays an important role in the formation of yield and quality improvement (Marschner, 2012, Oosterhuis et al., 2014). Potassium has strong mobility in plants and plays an important role in regulating cell osmotic pressure and balancing the cations and anions in the cytoplasm (Hu et al., 2016a). Phosphorus (P) is also an essential plant nutrient for various tree growth functions ( Jonard et al. 2015). Plants take up Phosphorus in its inorganic form as phosphate (Becquer et al., 2014). Phosphorus limitation decreases the efficiency of plant respiration (Jiang et al., 2019) and night respiration may increase along the N/P ratio. Therefore, considering the importance of the physiochemical properties of the soil mentioned above, the present study was carried out to perform a correlation and nutritional analysis of the soil under the populations of Grewia optiva Drummond in Himachal Pradesh.

\section{Materials and Methods}

\subsection{Study area and sampling station selection}

The present study on analysis of physiochemical characteristics of soil was carried out during May and November, 2019 and 2020 in seven districts of Himachal Pradesh i.e., Kangra, Mandi, Bilaspur, Solan, Sirmour, Una and Hamirpur, keeping in view the rich genetic diversity and phenotypically superior plant populations of Grewia optiva. Total thirty-five population (five population in each district) were selected. In each population six superior trees of $20 \mathrm{~cm}-30 \mathrm{~cm}$ diameter class were marked. The soil samples were collected from $15 \mathrm{~cm}-30$ $\mathrm{cm}$ depth underneath the selected populations of Grewia optiva Drummond. In each collection 35 composite soil samples (each a composite of six cores) were taken. The soil samples were collected in two consecutive years 2019 and 2020 , twice in year i.e., in the month of May and November. In 2019, first soil sample was collected during the Month of May, when this species was in leafing and flowering stage and second sample was taken at six months interval in the month of November, when the species was in the seed ripening and fodder lopping stage. Similarly, in the year 2020, two consecutive samples were collected in month of May and November from the same selected sites and depth $(15 \mathrm{~cm}-30$ $\mathrm{cm}$ ) respectively, from all thirty five populations. Thus, a total of four samples were taken in two consecutive years from each population and their data was statistically analysed separately for each interval. The pooled data from two years presented for statistical analysis and evaluation in the year 2021 under the present investigation. The morphometric traits of these selected superior trees have been recorded simultaneously. Further the correlation established between physiochemical characteristics of soil and morphometric traits of selected populations.

\subsection{Sampling procedure}

In order to collect soil samples (15 cm-30 cm depth) first removed grasses, litter and other plant residues from soil surface and collect soil samples by using soil collection tools. In each case, a triangular block was marked and soil samples were collected in plastic bags, which were sealed, and labelled properly. Collected soil were air dried, lightly grounded and sieved to remove coarse particles $(\geq 2 \mathrm{~mm})$ to ensure homogeneity. Mineral soil $<2 \mathrm{~mm}$ was subsampled in the field (approx. $150 \mathrm{~g}$ ) and transported to laboratory of Soil- science, University of Horticulture and Forestry, Nauni, Solan (HP) for further analysis of morphological (soil texture), physiochemical (Bulk density, $\mathrm{pH}, \mathrm{EC}$ ) and other available macronutrients $(\mathrm{N}, \mathrm{P}, \mathrm{K})$ on the basis of standard methods (Table 1).

\subsection{Analysis}

Analysis of variance (ANOVA) and Pearson correlation coefficient $(p<0.05)$ used to find correlation between physiochemical properties of soil and morphological characteristics of selected population of Grewia optiva which had been recorded simultaneously. Standard Soil Classification (Iram and Khan, 2018) (Table 2) has been used for the comparative analysis of different parameters of soil. 


\begin{tabular}{|c|c|c|}
\hline $\begin{array}{l}\text { Sl. } \\
\text { No. }\end{array}$ & Soil parameters & Analysis method used \\
\hline 1. & Soil texture & Robinson's pipette method \\
\hline 2. & $\mathrm{pH}$ & $\begin{array}{l}\text { Digital pH meter (Jackson, } \\
\text { 1973). }\end{array}$ \\
\hline 3. & Organic carbon (\%) & $\begin{array}{l}\text { Chromic acid titration method } \\
\text { (Walkley and Black, 1934) }\end{array}$ \\
\hline 4. & $\begin{array}{l}\text { Available nitrogen } \\
\left(\mathrm{kg} \mathrm{ha}^{-1}\right)\end{array}$ & $\begin{array}{l}\text { Micro Kjeldhal Method } \\
\text { (Subbaiah and Asija, 1956) }\end{array}$ \\
\hline 5. & $\begin{array}{l}\text { Available phosphorus } \\
\left(\mathrm{kg} \mathrm{ha}^{-1}\right)\end{array}$ & $\begin{array}{l}0.5 \mathrm{M} \text { sodium bicarbonate } \\
\left(\mathrm{NaHCO}_{3}\right) \text { at } 8.5 \mathrm{pH} \text { (Olsen et } \\
\text { al., 1954) }\end{array}$ \\
\hline 6. & Available K (kg ha $\left.{ }^{-1}\right)$ & $\begin{array}{l}\text { Flame photometric method ( } 1 \mathrm{~N} \\
\mathrm{NH}_{4} \mathrm{OAC} \text { extractable) (Merwin } \\
\text { and Peech, 1951) }\end{array}$ \\
\hline 7. & $\mathrm{EC}\left(\mathrm{dSm} \mathrm{m}^{-1}\right)$ & Digital EC meter (Jackson, 1973) \\
\hline
\end{tabular}

\section{Results and Discussion}

There was significant difference was observed in $\mathrm{pH}, \mathrm{OC}, \mathrm{EC}$, bulk density and in macronutrients $(N, P, K)$ within selected population of each district. Macro and Micro nutrients of the soil are equally important for growth and development of the plants but in the present investigation only the major macronutrients viz; N, P, K have been analyzed.

\section{1. $\mathrm{pH}$}

$\mathrm{pH}$ of the soil at depth of $15-30 \mathrm{~cm}$ of district Solan (Table 3) ranged from 6.70-7.66 with mean value of 7.24, of district Una ranged from 6.60-7.80 with mean value of 7.14, of Sirmour district ranged from $6.76-7.52$ with mean value 7.05 , of Hamirpur district ranged from 7.10-7.30 with mean value 7.16, Kangra district ranged from 6.01-7.65 with mean value of 6.84, of Mandi district ranged 5.1 to 6.3 with mean value 5.76 and of Bilaspur district ranged from 5.92 to 7.68 with mean value 7.12. The lowest $\mathrm{pH}$ of 5.1 was reported beneath Balt population of Mandi district, whereas highest beneath Nawami population of Una district. In this study $\mathrm{pH}$ range of moderately acidic to moderately alkaline reported best for the growth of this species. 100 leaf fresh weight, tree height, tree diameter, crown spread, no. of secondary branches and 100 fresh fruit weight showed positive correlation with the $\mathrm{pH}$ (Table 4, Figure 1). The most important role of $\mathrm{pH}$ is the control of nutrients solubility in soil. Nutrient availability usually decreases with increasing pH (Kazem et al., 2012).

\subsection{Electrical conductivity $\left(d S m^{-1}\right)(E C)$}

Electrical conductivity for the soil of district Solan (Table 3) ranged from $0.17-0.33\left(\mathrm{dSm}^{-1}\right)$ with mean value of $0.24(\mathrm{dSm}$ $\left.{ }^{1}\right)$, of district Una ranged from $0.11-0.22\left(\mathrm{dSm}^{-1}\right)$ with mean value of $0.11\left(\mathrm{dSm}^{-1}\right)$, District Sirmour 0.26-0.54 $\left(\mathrm{dSm}^{-1}\right)$ with

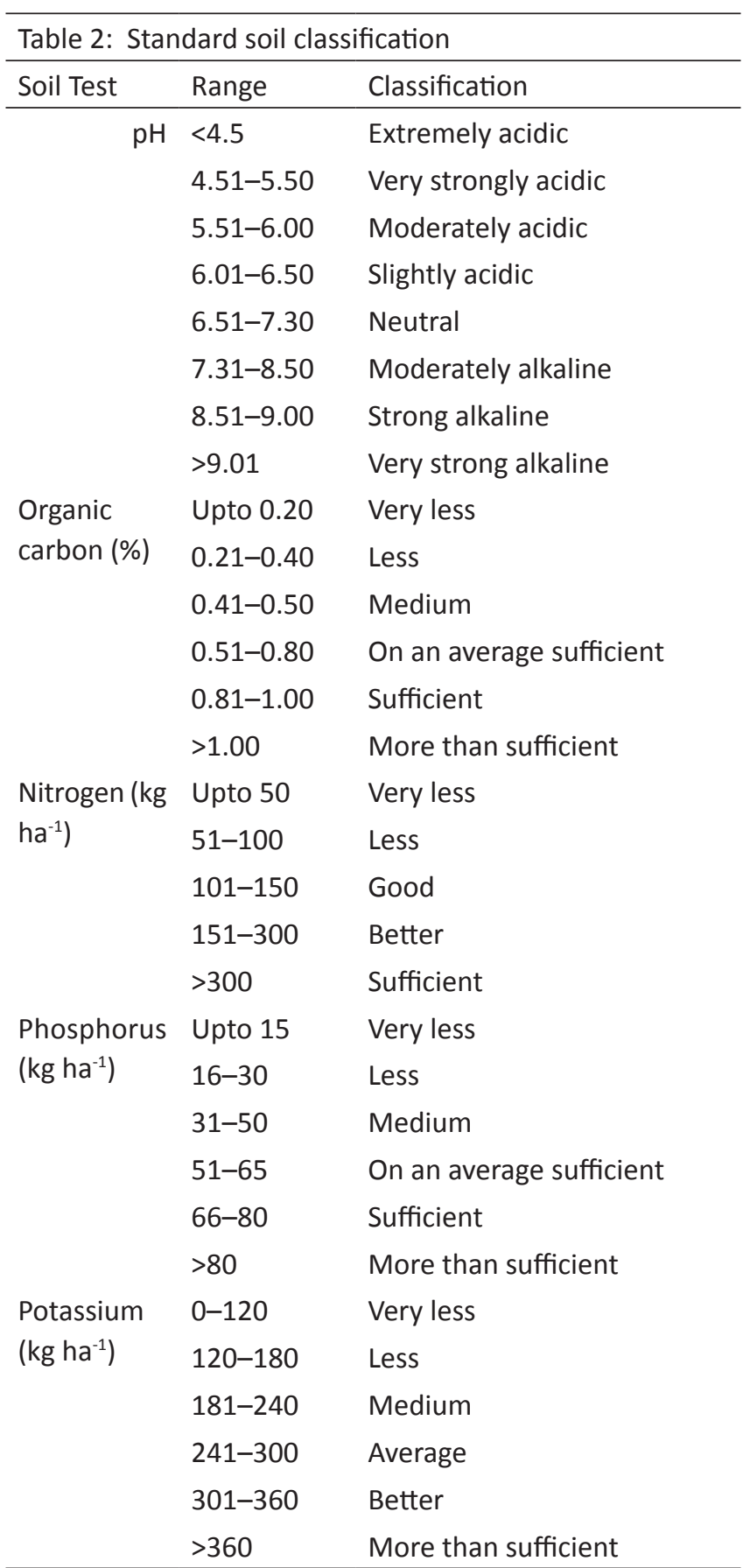

mean value of $0.42\left(\mathrm{dSm}^{-1}\right)$, of district Hamirpur 0.47-1.32 $\left(\mathrm{dSm}^{-1}\right)$ with mean value $0.85\left(\mathrm{dSm}^{-1}\right)$, District Kangra ranged between $0.11-0.19\left(\mathrm{dSm}^{-1}\right)$ with mean value 0.14 , of district mandi ranged $0.16-0.21$ with mean value $0.16\left(\mathrm{dSm}^{-1}\right)$ and of district Bilaspur $0.12-0.81\left(\mathrm{dSm}^{-1}\right)$ with mean value 0.39 $\left(\mathrm{dSm}^{-1}\right)$. The highest EC $1.32\left(\mathrm{dSm}^{-1}\right)$ was reported beneath Bhaleth population of Hamirpur district, whereas minimum $0.10\left(\mathrm{dSm}^{-1}\right)$ beneath old Kangra population. 100 leaf fresh weight, Tree diameter and crown spread showed moderately positive correlation with EC (Table 4, Figure 1). EC values affects uptake of nutrients by the tree. Very high or low EC 
Table 3: Physiochemical properties of Soils underneath different populations of Grewia optiva Drummond (Beul) of Himachal Pradesh at soil depth $15-30 \mathrm{~cm}$

\begin{tabular}{|c|c|c|c|c|c|c|c|c|}
\hline Populations & $\mathrm{pH}$ & $\begin{array}{c}\mathrm{EC} \\
\left(\mathrm{dSm^{-1 }}\right)\end{array}$ & $\begin{array}{c}\text { Organic } \\
\text { Carbon }\left(\mathrm{g} \mathrm{kg}^{-1}\right)\end{array}$ & $\begin{array}{c}\mathrm{N} \\
\left(\mathrm{kg} \mathrm{ha}^{-1}\right)\end{array}$ & $\begin{array}{c}\mathrm{P} \\
\left(\mathrm{kg} \mathrm{ha}^{-1}\right)\end{array}$ & $\begin{array}{c}\mathrm{K} \\
\left(\mathrm{kg} \mathrm{ha}^{-1}\right)\end{array}$ & Soil Texture & $\begin{array}{c}\text { Bulk } \\
\text { Density }\end{array}$ \\
\hline \multicolumn{9}{|l|}{ District- Solan } \\
\hline Kothi Kanwal & 7.32 & 0.25 & 7.40 & 295.83 & 14.93 & 240.05 & Sandy Loam & 1.14 \\
\hline Uncha Gaon & 7.54 & 0.19 & 10.70 & 343.92 & 30.61 & 282.67 & Clay loam & 1.13 \\
\hline Neri Kalan & 7.66 & 0.17 & 7.30 & 327.19 & 33.60 & 489.81 & Clay loam & 0.99 \\
\hline Sabathu & 6.70 & 0.33 & 6.60 & 278.06 & 23.89 & 360.88 & Sandy Loam & 1.17 \\
\hline Seri Devera & 7.02 & 0.30 & 3.50 & 270.79 & 17.92 & 212.80 & Clay loam & 1.16 \\
\hline Mean & 7.24 & 0.24 & 7.1 & 303.15. & 24.19 & 337.24 & & 1.11 \\
\hline SEm \pm & 0.09 & 0.01 & 0.56 & 6.54 & 2.27 & 12.62 & & 0.02 \\
\hline$C D(p=0.05)$ & 0.63 & 0.09 & 1.12 & 46.05 & 12.02 & 73.04 & & 0.05 \\
\hline \multicolumn{9}{|l|}{ District-Una } \\
\hline Kant & 6.60 & 0.14 & 10.02 & 280.36 & 25.60 & 285.38 & Gravelly Loam & 1.21 \\
\hline Nawami & 7.80 & 0.17 & 11.20 & 290.60 & 29.33 & 349.30 & Sandy Clay Loam & 1.04 \\
\hline Kharuni Bangana & 6.89 & 0.14 & 16.09 & 360.60 & 33.78 & 290.49 & Loam & 1.08 \\
\hline Badwar & 7.20 & 0.19 & 14.16 & 320.80 & 56.89 & 349.88 & Sandy Clay Loam & 1.18 \\
\hline Lamlehri & 7.22 & 0.22 & 10.08 & 280.18 & 46.70 & 477.21 & Gravelly Loam & 1.16 \\
\hline Mean & 7.14 & 0.11 & 12.31 & 307.60 & 38.47 & 350.45 & & 1.13 \\
\hline SEm \pm & 0.08 & 0.01 & 0.52 & 7.44 & 2.17 & 12.26 & & 0.01 \\
\hline$C D(p=0.05)$ & 0.88 & 0.05 & 1.09 & 55.04 & 11.89 & 77.8 & & 0.08 \\
\hline \multicolumn{9}{|l|}{ District- Sirmour } \\
\hline Machair & 6.76 & 0.36 & 14.65 & 378.45 & 57.12 & 439.28 & Silt Clay Loam & 1.70 \\
\hline Jajjer & 7.09 & 0.48 & 16.85 & 357.43 & 33.06 & 376.81 & Silt Loam & 1.24 \\
\hline Nehar Bagh & 7.52 & 0.54 & 19.05 & 379.51 & 49.40 & 512.46 & Loam & 1.39 \\
\hline Badon & 6.98 & 0.47 & 12.6 & 343.79 & 63.96 & 378.60 & Sandy Clay Loam & 1.08 \\
\hline Dhar Kyari & 6.91 & 0.26 & 15.75 & 368.22 & 35.68 & 285.96 & Sandy Clay Loam & 1.15 \\
\hline Mean & 7.05 & 0.42 & 15.78 & 365.48 & 47.84 & 398.62 & & 1.31 \\
\hline SEm \pm & 0.05 & 0.01 & 0.53 & 9.44 & 3.14 & 12.88 & & 0.02 \\
\hline$C D(p=0.05)$ & 0.54 & 0.17 & 2.83 & 33.05 & 23.01 & 84.04 & & 0.33 \\
\hline \multicolumn{9}{|l|}{ District- Hamirpur } \\
\hline Janhen & 7.30 & 0.47 & 17.21 & 370.50 & 43.60 & 240.43 & Sandy Clay Loam & 1.46 \\
\hline Jhinjhkari & 7.11 & 0.60 & 20.82 & 380.31 & 40.42 & 260.30 & Sandy Loam & 1.13 \\
\hline Harbal Neri & 7.10 & 1.15 & 27.25 & 372.13 & 35.46 & 272.10 & Sandy Loam & 1.11 \\
\hline Anu khurd & 7.20 & 0.82 & 25.06 & 344.91 & 37.90 & 212.66 & Gravelly Loam & 1.19 \\
\hline Bhaleth & 7.13 & 1.32 & 26.08 & 380.33 & 34.70 & 161.30 & Clay loam & 1.14 \\
\hline Mean & 7.16 & 0.85 & 23.28 & 369.63 & 38.35 & 229.35 & & 1.14 \\
\hline SEm \pm & 0.06 & 0.02 & 0.62 & 11.34 & 2.19 & 10.62 & & 0.01 \\
\hline$C D(p=0.05)$ & 1.02 & 0.20 & 5.75 & 25.03 & 13.05 & 54.08 & & 0.09 \\
\hline \multicolumn{9}{|l|}{ District - Kangra } \\
\hline Katoi & 6.65 & 0.15 & 9.90 & 282.24 & 24.64 & 283.36 & Sandy, Clay, Loam & 1.21 \\
\hline
\end{tabular}




\begin{tabular}{|c|c|c|c|c|c|c|c|c|}
\hline Populations & $\mathrm{pH}$ & $\begin{array}{c}\mathrm{EC} \\
\left(\mathrm{dSm^{-1 }}\right) \\
\end{array}$ & $\begin{array}{c}\text { Organic } \\
\text { Carbon }\left(\mathrm{g} \mathrm{kg}^{-1}\right)\end{array}$ & $\begin{array}{c}\mathrm{N} \\
\left(\mathrm{kg} \mathrm{ha}^{-1}\right)\end{array}$ & $\begin{array}{c}\mathrm{P} \\
\left(\mathrm{kg} \mathrm{ha}^{-1}\right)\end{array}$ & $\begin{array}{c}\mathrm{K} \\
\left(\mathrm{kg} \mathrm{ha}^{-1}\right)\end{array}$ & Soil Texture & $\begin{array}{c}\text { Bulk } \\
\text { Density }\end{array}$ \\
\hline Baluglua & 7.65 & 0.16 & 11.25 & 291.64 & 29.12 & 348.32 & Loam & 1.08 \\
\hline Old Kangra & 6.71 & 0.10 & 10.05 & 363.77 & 33.60 & 284.48 & Loam & 1.15 \\
\hline Dohan & 6.01 & 0.11 & 14.10 & 319.87 & 53.76 & 349.44 & Gravelly Loam & 1.13 \\
\hline Balla & 7.21 & 0.19 & 7.05 & 272.83 & 44.80 & 577.92 & Sandy Loam & 1.22 \\
\hline Mean & 6.84 & 0.14 & 10.47 & 306.07 & 37.18 & 368.70 & & 1.15 \\
\hline SEm \pm & 0.04 & 0.01 & 0.41 & 8.34 & 2.07 & 11.22 & & 0.02 \\
\hline$C D(p=0.05)$ & 0.82 & 0.07 & 1.58 & 56.02 & 12.86 & 82.05 & & 0.09 \\
\hline \multicolumn{9}{|c|}{ District - Mandi } \\
\hline Patta & 5.7 & 0.18 & 2.70 & 231 & 37.5 & 316 & Clay Loam & 1.15 \\
\hline Gangal & 5.4 & 0.21 & 1.01 & 132 & 35.5 & 480 & Loam & 1.17 \\
\hline Bagla & 6.3 & 0.12 & 1.48 & 115 & 18.8 & 182 & Sandy Loam & 1.38 \\
\hline Balt & 5.1 & 0.15 & 1.10 & 134 & 35.8 & 258 & Sandy clay Loam & 1.01 \\
\hline Bharnoi & 6.3 & 0.18 & 2.63 & 136 & 18.8 & 414 & Sandy clay Loam & 1.30 \\
\hline Mean & 5.76 & 0.16 & 1.78 & 149.5 & 29.28 & 330 & & 1.20 \\
\hline SEm \pm & 0.04 & 0.02 & 0.09 & 3.54 & 1.17 & 10.32 & & 0.01 \\
\hline $\mathrm{CD}(p=0.05)$ & 0.6 & 0.09 & 0.17 & 53.01 & 15.60 & 72.06 & & 0.034 \\
\hline \multicolumn{9}{|c|}{ District -Bilaspur } \\
\hline Ghumarwin & 7.38 & 0.33 & 22.10 & 362.70 & 42 & 246.70 & Clay Loam & 1.33 \\
\hline Barthin & 7.68 & 0.25 & 27.50 & 419.20 & 45.62 & 328.80 & Loam & 1.20 \\
\hline Kuthera & 5.92 & 0.12 & 15.20 & 378.32 & 45.20 & 213.40 & Sandy Loam & 1.18 \\
\hline Nehari & 7.26 & 0.81 & 17.21 & 364.40 & 37.20 & 297.80 & Clay Loam & 1.08 \\
\hline Jukhala & 7.40 & 0.46 & 27.12 & 386.30 & 38.10 & 279.84 & Sandy Loam & 1.22 \\
\hline Mean & 7.12 & 0.39 & 21.82 & 382.18 & 41.62 & 267.30 & & 1.18 \\
\hline SEm \pm & 0.09 & 0.01 & 0.76 & 11.84 & 2.31 & 7.72 & & 0.02 \\
\hline$C D(p=0.05)$ & 0.60 & 0.08 & 6.76 & 42.09 & 5.80 & 37.60 & & 0.063 \\
\hline
\end{tabular}

decreases plants fresh weight (FW) and dry weight (DW), leaf size, leaf water content, leaf net photosynthetic rate $\left(P_{n}\right)$, stomatal conductance $\left(G_{s}\right)$, transpiration rate $\left(T_{r}\right)$, (Sonneveld and De Kreij, 1996). In present study EC recorded above 0.6 $\left(\mathrm{dSm}^{-1}\right)$ up to $1.32\left(\mathrm{dSm}^{-1}\right)$ (Table 3 ) lies in range of non-saline soil (NRCS-USDA,1999) which was recorded favorable for growth of this species.

\subsection{Organic carbon $\left(\mathrm{g} \mathrm{kg}^{-1}\right)(O C)$}

Organic carbon for the soil of district Solan (Table 3) ranged from 3.50-10.70 $\left(\mathrm{g} \mathrm{kg}^{-1}\right)$ or $0.3-1.07 \%$ with mean value of $7.1\left(\mathrm{~g} \mathrm{~kg}^{-1}\right) / 0.7 \%$, of district Una ranged from 10.02-16.09 $\left(\mathrm{g} \mathrm{kg}^{-1}\right)$ or $1.02-1.609 \%$ with mean value of $12.31\left(\mathrm{~g} \mathrm{~kg}^{-1}\right)$ or $1.23 \%$, District Sirmour $12.6-19.05\left(\mathrm{~g} \mathrm{~kg}^{-1}\right)$ or $1.26-1.905 \%$ with mean value of $15.78\left(\mathrm{~g} \mathrm{~kg}^{-1}\right) / 1.57 \%$, of district Hamirpur $17.21-27.25\left(\mathrm{~g} \mathrm{~kg}^{-1}\right)$ or $1.72-2.72 \%$ with mean value of 23.28 $\left(\mathrm{g} \mathrm{kg}^{-1}\right) / 2.3 \%$, District Kangra ranged between $7.05-14.10$ (g $\left.\mathrm{kg}^{-1}\right)$ or $0.70-1.41 \%$ with mean value $10.47\left(\mathrm{~g} \mathrm{~kg}^{-1}\right) / 1.04 \%$, of district Mandi ranged 1.01-2.70 $\left(\mathrm{g} \mathrm{kg}^{-1}\right)$ or $0.10-0.27 \%$ with mean value $1.78\left(\mathrm{~g} \mathrm{~kg}^{-1}\right) / 0.17 \%$ and of district Bilaspur $15.20-27.50\left(\mathrm{~g} \mathrm{~kg}^{-1}\right)$ or $1.52-2.75 \%$. The highest organic carbon recorded in soil of Barthin Population of Bilaspur district, whereas lowest found in Gangal Population of Mandi district. 100 leaf fresh weight, 100 leaf dry weight, and crown spread showed highly positive correlation with OC, whereas leaf area, tree diameter and tree height showed negative correlation with it (Table 4, Figure 1). Soil organic carbon is a natural resource for the sustainable development of human society and a key foundation for sustainable forestry development (Pan et al., 2015). It plays an important role in the formation and conservation of soil structure, soil nutrient cycling and soil biodiversity.

\subsection{Nitrogen $\left(\mathrm{kg} \mathrm{ha}^{-1}\right)$}

Nitrogen underneath district Solan (Table 3) population ranged from 270.79-343.92 ( $\left.\mathrm{kg} \mathrm{ha}^{-1}\right)$ with mean value of $303.15\left(\mathrm{~kg} \mathrm{ha}^{-1}\right)$, of district Una ranged from 280.18-360.60 $\left(\mathrm{kg} \mathrm{ha}^{-1}\right)$ with mean value of $307.60\left(\mathrm{~kg} \mathrm{ha}^{-1}\right)$, District Sirmour 
Table 4: Correlation between growth characteristics of selected populations of Grewia optiva Drummond and soil physiochemical characteristics underneath these selected populations

\begin{tabular}{|c|c|c|c|c|c|c|c|c|c|}
\hline & LA & 100LFW & 100LDW & $\mathrm{TH}$ & TD & CS & NPB & NSB & 100FFW \\
\hline LA & 1 & & & & & & & & \\
\hline 100LFW & 0.239 & 1 & & & & & & & \\
\hline 100LDW & 0.087 & 0.438 & 1 & & & & & & \\
\hline $\mathrm{TH}$ & -0.110 & -0.052 & -0.056 & 1 & & & & & \\
\hline TD & -0.090 & 0.060 & 0.305 & 0.562 & 1 & & & & \\
\hline CS & 0.289 & -0.004 & 0.121 & 0.036 & 0.188 & 1 & & & \\
\hline NPB & 0.094 & -0.037 & 0.122 & 0.213 & $0.260^{*}$ & 0.409 & 1 & & \\
\hline NSB & 0.053 & -0.086 & 0.078 & 0.327 & $0.364^{*}$ & 0.363 & $0.697^{*}$ & 1 & \\
\hline 100FFW & -0.090 & 0.160 & 0.154 & 0.352 & 0.186 & -0.004 & 0.161 & 0.179 & 1 \\
\hline 100FDW & -0.029 & -0.192 & 0.201 & 0.241 & 0.239 & -0.004 & $0.256^{*}$ & 0.435 & 0.152 \\
\hline $\mathrm{pH}$ & -0.069 & 0.059 & 0.218 & 0.227 & 0.320 & 0.235 & 0.152 & 0.219 & 0.300 \\
\hline EC & 0.095 & $0.200^{*}$ & 0.045 & 0.089 & 0.141 & 0.118 & -0.217 & -0.312 & -0.063 \\
\hline $\mathrm{OC}$ & 0.171 & $0.392^{*}$ & $0.285^{*}$ & 0.138 & 0.191 & $0.292^{*}$ & 0.001 & -0.171 & 0.104 \\
\hline$P$ & $0.448^{*}$ & 0.140 & -0.215 & -0.105 & -0.099 & $0.303^{*}$ & 0.182 & -0.129 & -0.161 \\
\hline $\mathrm{N}$ & $0.323^{*}$ & $0.366^{*}$ & $0.343^{*}$ & -0.031 & -0.023 & $0.385^{*}$ & $0.270^{*}$ & -0.104 & $0.251^{*}$ \\
\hline K & 0.173 & -0.035 & -0.284 & -0.358 & -0.398 & 0.0457 & -0.013 & -0.03 & -0.00 \\
\hline BD & 0.049 & -0.243 & -0.239 & 0.076 & -0.010 & -0.006 & $0.397^{*}$ & 0.184 & -0.058 \\
\hline
\end{tabular}

Table 4: Continue...

\begin{tabular}{|c|c|c|c|c|c|c|c|c|}
\hline & 100FDW & $\mathrm{pH}$ & EC & $\mathrm{OC}$ & $\mathrm{P}$ & $\mathrm{N}$ & $\mathrm{K}$ & $\mathrm{BD}$ \\
\hline \multicolumn{9}{|l|}{ LA } \\
\hline \multicolumn{9}{|l|}{ 100LFW } \\
\hline \multicolumn{9}{|l|}{ 100LDW } \\
\hline \multicolumn{9}{|l|}{ TH } \\
\hline \multicolumn{9}{|l|}{ TD } \\
\hline \multicolumn{9}{|l|}{ CS } \\
\hline \multicolumn{9}{|l|}{ NPB } \\
\hline \multicolumn{9}{|l|}{ NSB } \\
\hline \multicolumn{9}{|l|}{ 100FFW } \\
\hline 100FDW & 1 & & & & & & & \\
\hline $\mathrm{pH}$ & -0.018 & 1 & & & & & & \\
\hline EC & -0.398 & 0.278 & 1 & & & & & \\
\hline OC & -0.343 & 0.269 & 0.687 & 1 & & & & \\
\hline $\mathrm{P}$ & -0.263 & 0.064 & 0.115 & 0.255 & 1 & & & \\
\hline $\mathrm{N}$ & -0.232 & 0.099 & 0.313 & 0.629 & 0.303 & 1 & & \\
\hline K & -0.08 & 0.048 & -0.199 & -0.389 & 0.269 & -0.134 & 1 & \\
\hline$B D$ & 0.147 & -0.120 & -0.426 & -0.365 & 0.107 & -0.115 & 0.201 & 1 \\
\hline
\end{tabular}

Correlation Significant at $p<0.05$; LA: leaf area, 100 LFW: 100 leaf fresh weight, 100 LDW: 100 leaf dry weight, TH: tree height, TD: tree diameter, CS: crown spread, NPB: no. of primary branches, NSB: no. of secondary branches, 100 FFW: 100 fresh fruit weight, 100 FDW: 100 fruit Dry weight, EC: electrical conductivity, OC: organic carbon, P: phosphorus, N: nitrogen, K: potassium, BD: bulk density 


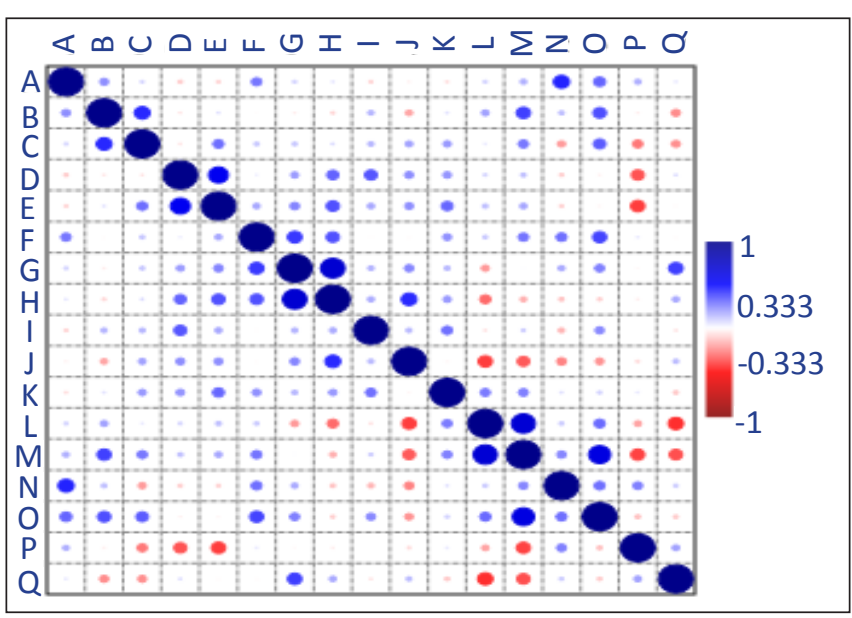

Figure 1: Graphical representation of correlation between growth characteristics of selected populations of Grewia optiva Drummond and soil physiochemical characteristics underneath these selected populations. Where, (A) LA: leaf area, (B) 100 LFW: 100 leaf fresh weight, (C) 100 LDW: 100 leaf dry weight, (D) TH: tree height, (E) TD: tree diameter, (F) CS: crown spread, (G) NPB: no. of primary branches, (H) NSB: no. of secondary branches, (I) $100 \mathrm{FFW}: 100$ fresh fruit weight, (J) 100 FDW: 100 fruit Dry weight, (K) pH, (L) EC: electrical conductivity, (M) OC: organic carbon, (N) P: phosphorus, (O) $\mathrm{N}$ : nitrogen, $(\mathrm{P}) \mathrm{K}$ : potassium, (Q) BD: bulk density

$343.79-379.51\left(\mathrm{~kg} \mathrm{ha}^{-1}\right)$ with mean value of 365.48 (kg ha $\left.{ }^{1}\right)$, of district Hamirpur 344.91-380.33 $\left(\mathrm{kg} \mathrm{ha}^{-1}\right)$ with mean value of $369.63\left(\mathrm{~kg} \mathrm{ha}^{-1}\right)$, District Kangra ranged between 272.83-363.77 ( $\left.\mathrm{kg} \mathrm{ha}^{-1}\right)$ with mean value $306.07\left(\mathrm{~kg} \mathrm{ha}^{-1}\right)$, of district Mandi ranged between $115-231\left(\mathrm{~kg} \mathrm{ha}^{-1}\right)$ with mean value $149.5\left(\mathrm{~kg} \mathrm{ha}^{-1}\right)$ and of district Bilaspur ranged between $362.70-419.20\left(\mathrm{~kg} \mathrm{ha}^{-1}\right)$ with mean value $382.18\left(\mathrm{~kg} \mathrm{ha}^{-1}\right)$. The highest Nitrogen recorded in soil of Barthin Population of Bilaspur district, whereas lowest found underneath Bagla Population of Mandi district. Leaf area, 100 leaf fresh weight, 100 leaf dry weight, crown spread, no. of primary branches and 100 fruit fresh weight showed positive correlation with the available nitrogen in the soil (Table 4, fig 1). Similar results recorded in Douglas-fir by (Brix and Ebell,1969), which revealed that fertilization with Nitrogen increased basal area increment, stem height, and branch length, leaf area, leaf length-width, the no. of leaves per shoot increased markedly. (Wang et al.,2012) reported increased specific leaf area (SLA) and leaf area index (LAI) with $\mathrm{N}$ fertilization. A study on Fagus sylvatica by (Meier and Leuschner, 2008) reported positive effects on leaf area and LAI in forests with increased in nitrogen availability.

\subsection{Phasphorus $\left(\mathrm{kg} \mathrm{ha}^{-1}\right)$}

Phosphorus content in soil of district Solan (Table 3 ) ranged from 14.93-33.60 $\left(\mathrm{kg} \mathrm{ha}^{-1}\right)$ with mean value of $24.19(\mathrm{~kg}$ $\left.\mathrm{ha}^{-1}\right)$, of district Una ranged from $25.60-56.89\left(\mathrm{~kg} \mathrm{ha}^{-1}\right)$ with mean value of $38.47\left(\mathrm{~kg} \mathrm{ha}^{-1}\right)$, District Sirmour $\left(\mathrm{kg} \mathrm{ha}^{-1}\right)$ ranged between 33.06-63.96 $\left(\mathrm{kg} \mathrm{ha}^{-1}\right)$ with mean value of $47.84(\mathrm{~kg}$ $\left.\mathrm{ha}^{-1}\right)$, of district Hamirpur 34.70-43.60 $\left(\mathrm{kg} \mathrm{ha}^{-1}\right)$ with mean value of $38.35\left(\mathrm{~kg} \mathrm{ha}^{-1}\right)$, District Kangra ranged between 24.64-53.76 $\left(\mathrm{kg} \mathrm{ha}^{-1}\right)$ with mean value $37.18\left(\mathrm{~kg} \mathrm{ha}^{-1}\right)$, of district Mandi ranged between 18.8-37.5 $\left(\mathrm{kg} \mathrm{ha}^{-1}\right)$ with mean value $29.28\left(\mathrm{~kg} \mathrm{ha}^{-1}\right)$ and of district Bilaspur ranged between $37.20-45.62\left(\mathrm{~kg} \mathrm{ha}^{-1}\right)$ with mean value $41.62\left(\mathrm{~kg} \mathrm{ha}^{-1}\right)$. The highest available phosphorus recorded in soil of Badon Population of Sirmour district, whereas lowest underneath Bharnoi Population of Mandi district. Leaf area and crown spread showed positive correlation (Table 4, fig 1) with the available phosphorus in soil. In a similar study by (Herbert and Fownes, 1995) in native Metrosideros polymorpha forest showed that increased available phosphorus promoted an increase in photosynthetic area which led to increased tree growth.

\subsection{Potassium $\left(\mathrm{kg} \mathrm{ha}^{-1}\right)$}

Potassium content in soil of district Solan (Table 3) ranged from 212.80-489.81 $\left(\mathrm{kg} \mathrm{ha}^{-1}\right)$ with mean value of 337.24 $\left(\mathrm{kg} \mathrm{ha}^{-1}\right)$, of district Una ranged from 285.38-477.21 (kg ha-1) with mean value of $350.45\left(\mathrm{~kg} \mathrm{ha}^{-1}\right)$, District Sirmour $\left(\mathrm{kg} \mathrm{ha}^{-1}\right)$ ranged between 285.96-512.46 ( $\left.\mathrm{kg} \mathrm{ha}^{-1}\right)$ with mean value of $398.62\left(\mathrm{~kg} \mathrm{ha}^{-1}\right)$, of district Hamirpur 161.30-272.10 (kg ha-1) with mean value of $229.35\left(\mathrm{~kg} \mathrm{ha}^{-1}\right)$, District Kangra ranged between 283.36-577.92 $\left(\mathrm{kg} \mathrm{ha}^{-1}\right)$ with mean value 368.70 $\left(\mathrm{kg} \mathrm{ha}^{-1}\right)$, of district Mandi ranged between 182-480 $\left(\mathrm{kg} \mathrm{ha}^{-1}\right)$ with mean value $330\left(\mathrm{~kg} \mathrm{ha}^{-1}\right)$ and of district Bilaspur ranged between $213.40-328.80\left(\mathrm{~kg} \mathrm{ha}^{-1}\right)$ with mean value 267.30 ( $\mathrm{kg}$ $\left.\mathrm{ha}^{-1}\right)$. The highest available Pottasium recorded underneath Balla of Population of Kangra district, whereas lowest Bharnoi Population of Mandi district. Leaf area showed moderately positive correlation with the available potassium in the soil (Table 4, figure 1). A similar study in Eucalyptus grandis by (Battie-Laclau et al., 2013) reported that $\mathrm{K}$ and $\mathrm{Na}$ applications enhanced tree leaf area by increasing both leaf longevity and the mean area of individual leaves.

\subsection{Soil Texture}

Soil of different texture were founded underneath populations of different districts (Table 3 ) viz; $50 \%$ of soil texture observed as sandy loamy and sandy clay loamy followed by clay loamy $(20 \%)$, gravelly loamy $(10 \%)$, loamy $(10 \%)$ and remaining silty clay loamy $(5 \%)$ and silty loam (5\%). The tree has a capacity to grow in almost any type of soil but sandy loam with proper moisture is most suitable for its proper growth. (Singh et al., 2018).

\subsection{Bulk density (BD)}

Bulk density underneath soil of district Solan populations (Table 3) ranged from $0.99-1.17$ with mean value of 1.11, of district Una ranged from 1.04-1.21 with mean value of 1.13, district Sirmour ranged between 1.15-1.70 with mean value of 1.31, of district Hamirpur 1.11-1.46 with mean value of 1.14, District Kangra ranged between 1.08-1.22 with mean value 1.15, of district Mandi ranged between 1.01-1.38 with 
mean value 1.20 and of district Bilaspur ranged between 1.08-1.33 with mean value 1.18. The highest BD recorded underneath Machair Population of Sirmour district, whereas lowest in soil of Neri Kalan population of Solan district. No. of primary branches showed highly positive correlation with the $B D$, no. of secondary branches, 100 fruit dry weight showed moderately positive correlation with the BD (Table 4, Figure 1). The bulk density depends on compaction, consolidation of the soil but it is negatively correlated to the organic content (Micheni et al., 2004).

Analysis of physiochemical characteristics of soil underneath selected Grewia optiva Drummond populations of Himachal Pradesh concluded that this species can grow in moderately acidic to moderately alkaline $\mathrm{pH}$. Soil which was rich in $\mathrm{OC}$ gave more growth in leaf area, 100 leaf dry weight and in crown spread. Increase in available nitrogen positively increase vegetative growth of the trees. Available phosphorus was present in less and in medium concentration in all the soil samples except Badon population of Sirmour district. Whereas potassium was present in medium, average, better and in more than sufficient amount underneath selected populations at different districts but the soil underneath Bhaleth population of Hamirpur district found less in available potassium. Nitrogen is considered to be the most important nutrient, and plants absorb more nitrogen than any other element. Nitrogen is essential in the formation of protein, and protein makes up much of the tissues of most living things. Increase in available phosphorus affected positively leaf area, crown spread, no. of branches and fruit. Phosphorus, is linked to a plant's ability to use and store energy, including the process of photosynthesis. It's also needed to help plants grow and develop normally (Yosuf et al., 2017). Potassium is known to affect cell division, cell permeability formation of carbohydrates, translocation of sugars, various enzyme actions and resistance of some plants to certain diseases (Miller and Turk, 2002). It helps strengthen plants' abilities to resist disease and plays an important role in increasing crop yields and overall quality. Potassium also protects the plant when the weather is cold or dry, strengthening its root system and preventing wilt. Therefore Nitrogen (promotes leaf growth), Phosphorus (root, flower, and fruit), and Potassium supports stem and root growth and protein analysis. Variability observed in soil texture, which concluded that this species can grow on variety of soil but maximum growth observed under Sandy loam soil. Variability in soil texture may contribute to the variation in nutrient storage and availability, water retention and transport and binding and stability of soil aggregates. Soil texture directly or indirectly influences soil functions such as soil erosion, water availability (Adhikari et al., 2009).

\section{Conclusion}

Study concludes that this species can grow in moderately acidic to moderately alkaline $\mathrm{pH}$. Thirty biologically screened populations found good in soil fertility status under crop land situation for the production of quality planting material except populations of Mandi district. Fifty percent of soil texture examined as sandy loamy and sandy clay loamy underneath these populations which favours the growth of this species. Screened populations under study have been found as best seed sources to obtain highly nutritive strains.

\section{Acknowledgment}

The authors are very thankful to National Mission on Himalayan Studies (NMHS) G.B. Pant National Institute of Himalayan Environment and Sustainable Development (GBPNIHESD), Kosi-Katarmal, Almora-263643, Uttarakhand, India under the Ministry of Environment Forest \& Climate Change, Govt. of India, Jorbhag Road, New Delhi-110003 for funding this project entitled "Biological Screening, Conservation and Establishment of Gene Bank of Grewia optiva Drummond (Beul)." to this department for undertaking this present study.

\section{References}

Adhikari, K., Guadagnini, A., Toth, G., Hermann, T., 2009. Geostatistical analysis of surface soil texture from Zala County in western Hungary. International Symposium on Environment, Energy and Water in Nepal: Recent Researches and Direction for Future, 219-224.

Bai, L., Deng, H., Zhang, X., Yu, X., Li, Y., 2016. Gibberellin Is Involved in Inhibition of Cucumber Growth and Nitrogen Uptake at Suboptimal Root-Zone Temperatures. PLoS One 11(5), e0156188. https://doi.org/10.1371/journal. pone.0156188

Battie-Laclau, P., Laclau, J.P., Piccolo, M.C., Arenque Beri , C., Mietton, L., Muniz, M.R., Meille- Buckeridge, M.S., Nouvellon, Y., Ranger, J., Bouillet, J.P., 2013. Influence of Potassium and Sodium Nutrition on Leaf Area Components in Eucalyptus grandis Trees. Plant and Soil 371, 19-35.

Becquer, A., Trap, J., Irshad, U., Ali, M.A., Claude, P., 2014. From soil to plant, the journey of $P$ through trophic relationships and ectomycorrhizal association. Frontiers in Plant Science 5, 548. doi: 10.3389/fpls.2014.00548

Bhagta.S., Thakur, P., Sharma D., 2021. Genetic divergence study in Grewia optiva through Quantitative and Molecular markers. International Journal of Economic Plants, 029033. DOI: HTTPS://DOI.ORG/10.23910/2/2020.0396

Brix, H., Ebell, L.F., 1969. Effects of Nitrogen fertilization on Growth, leaf area, and photosynthesis rate in DouglasFir. Forest Sciences 15, 189-196.

Chen, L.H., Dong, T.F., Duan, B.L., 2014. Sex-specific carbon and nitrogen partitioning under $\mathrm{N}$ deposition in Populus cathayana. Trees 28, 793-806.https://doi.org/10.1007/ s00468-014-0992-3.

Drake, J.E., Gallet-Budynek, A., Hofmockel, K.S., Bernhardt, E.S., Billings, S.A., Jackson, R.B., 2013. Increases in the flux of carbon belowground stimulate nitrogen uptake 
and sustain the long-term enhancement of forest productivity under elevated $\mathrm{CO}_{2}$. Ecology Letters 14, 349-357. doi: 10.1111/j.1461-0248.2011. 01593.x

Ellsworth, D.S., Anderson, I.C., Crous, K.Y., Cooke, J., Drake, J.E., Gherlenda, A.N., 2017. Elevated $\mathrm{CO}_{2}$ does not increase Eucalypt forest productivity on a lowphosphorus soil. Nature Climate Change 7, 279-283. doi: $10.1038 /$ nclimate3235

Herbert, D.A., Fownes, J.H., 1995. Phosphorus limitation of forest leaf area and net primary production on a highly weathered soil. Biogeochemistry 29, 223-235.

Hu, W., Zhao, W., Yang, J., Oosterhuis, D.M., Loka, D.A., Zhou, Z., 2016a. Relationship between potassium fertilization and nitrogen metabolism in the leaf subtending the cotton (Gossypium hirsutum L.) boll during the boll development stage. Plant Physiology and Biochemistry 101, 113-123. doi: 10.1016/j. plaphy.2016.01.019

Iram, A., Khan, T., 2018. Analysis of soil quality using physicochemical parameters with special emphasis on fluoride from selected sites of sawai Madhopur Tehsil, ajasthan. International Journal of Environmental Sciences and Natural Resources 12(5), 555847

Jackson, M.L., 1973. Soil Chemical Analysis. Prentice Hall of India (Pvt.) Ltd., New Delhi. 85, 251-252.

Ji, D.H., Mao, Q.Z., Watanabe, Y., Kitao, M., Kitaoka, S., 2015. Effect of nitrogen loading on the growth and photosynthetic responses of Japanese larch seedlings grown under different light regimes. Journal of Agricultural Meteorology 71, 232-238. https://doi. org/10.2480/ agrmet. D-14-00027

Jiang, M., Caldararu, S., Zaehle, S., Ellsworth, D.S., Medlyn, B.E., 2019. Towards a more physiological representation of vegetation phosphorus processes in land surface models. New Phytologist 222, 1223-1229. doi: 10.1111/ nph.15688

Jonard, M., Furst, A., Verstraeten, A., 2015. Tree mineral nutrition is deteriorating in Europe. Global Change Biology 21, 418-430.

Katoch, R., Singh, S.K., Tripath, A., Kumar, N., 2017. Effect of seasonal variation in biochemical composition of leaves of fodder trees prevalent in the mid-hill region of Himachal Pradesh. Range Management and Agroforestry 38, 234-240.

Kazem, H., Tayebeh, M., Farani, S., Jamaati-e-Somarin, 2012. Effect of elemental sulphur and compost on $\mathrm{pH}$, electrical conductivity and phosphorus availability of one clay soil. African Journal of Biotechnology 11, 1425-1432.

Liu, N., Wang, J., Guo, Q., Wu, S., Rao, X., Cai, X., Lin, Z., 2018. Alterations in leaf nitrogen metabolism indicated the structural changes of subtropical forest by canopy addition of nitrogen. Ecotoxicology and Environmental Safety 160, 134-143. https://doi.org/10.1016/j. ecoenv.2018.05.037 (2018)
Marschner, H., 2012. Marschner's Mineral Nutrition of Higher Plants. Cambridge, MA: Academic press.

Meier, I.C., Finzi, A.C., Phillips, R.P., 2017. Root exudates increase $\mathrm{N}$ availability by stimulating microbial turnover of fast-cycling N pools. Soil Biology and Biochemistry 106, 119-128. doi: 10.1016/j.soilbio.2016.12.004

Meier, I.C., Leuschner, C., 2008. Leaf Size and Leaf Area Index in Fagus sylvatica Forests: Competing Effects of Precipitation, Temperature, and Nitrogen Availability. Ecosystem 11, 655-669

Merwin, H.D., Peech, M., 1951. Exchangeability of soil potassium in the sand, silt and clay fractions as influenced by the nature of the complementary exchangeable cation. Soil Science Society of America Journal. 15, 125-128.

Micheni, A., Kihanda, F., Irungu, J., 2004. Soil organic matter (SOM): The basis for improved crop production in arid and semi-arid climate of eastern Kenya. In Managing Nutrient Cycles to Sustain Soil Fertility in Sub-Saharan Africa; Bationo, A., Ed.; Academy Science Publisher: Nairobi, Kenya, 608

Miller, C., Turk, L.M., 2002. Fundamentals of soil science Biotech. Books, 1123/74, Trinagar, Delhi, India pp. 157

Mukherjee, A., Modal, T., Bist, J.K., Pattanayak, A., 2018. Farmer's preference of fodder trees in mid hills of Uttarakhand: A comprehensive ranking using analytical hierarchy process. Range Management and Agroforestry 39, 115-120.

NRSC-USDA, 1999. Soil Taxonomy - A Basic System of Soil Classification for Making and Interpreting Soil Surveys. Agriculture Handbook. No., 436.

Ochoa-Hueso, R., Hughes, J., Delgado-Baquerizo, M., Drake, J.E., Tjoelker, M.G., Pineiro, J., 2017. Rhizospheredriven increase in nitrogen and phosphorus availability under elevated atmospheric $\mathrm{CO}_{2}$ in a mature Eucalyptus woodland. Plant and Soil 416, 283-295. doi: 10.1007/s11104-017-3212-2

Olsen, S.R., Cole, C.V., Watanable, F.S., Dean, L.A., 1954. Estimation of available phosphorus in soil by extraction with $\mathrm{NaHCO}_{3}$, USDA Circular, US Washington. 939, 19p.

Oosterhuis, D., Loka, D., Kawakami, E., Pettigrew, W., 2014. The physiology of potassium in crop production. Advances in Agronomy 126, 203-234. doi: 10.1016/B978-0-12800132-5.00003-1

Pan, G., Lu, H., Li, L., Zhang, J., Zhang, X., 2015. Soil Carbon Sequestration with Bioactivity: A New Emerging Frontier for Sustainable Soil Management. Advances in Earth Science 30, 940-951.

Schleppi, P., Korner, C., Klein, T., 2019. Increased Nitrogen availability in the soil under mature Picea abies trees exposed to elevated $\mathrm{Co}_{2}$ concentrations. Frontiers in Forests and Global Change 2, 59.

Singh, B., Makkar, P.S., Negi, S.S., 1989. Rate and extent of digestion and potentially digestible dry matter and cell 
wall of various tree leaves. Journal of Dairy Science 72, 3233-3239.

Singh, C., Singh, R., Himshikha, 2018. Grewia optiva (Drumm. Ex Burr)-A Multi-Purpose Tree Under Agroforestry in Sub-Tropical Region of Western Himalaya. Journal of Tree Sciences 37, 36-43.

Smith, J.L., Doran, J.W., 1996. Measurement and use of $\mathrm{pH}$ and electrical conductivity for soil quality analysis. Methods for assessing soil quality, 169-185.

Sonneveld, C., De Kreij, C., 1996. Response of cucumber (Cucumis sativus L.) to an unequal distribution of salts in the root environment. Plant and Soil 209, 45-56.

Subbaiah, B.V., Asija, G.L., 1956. A rapid method for the estimation of available nitrogen in soil. Current Science 25, 258-260.

Sumithra, S., Ankalaiah, C., Rao, D., Yamuna, R.T., 2013. A case study on physico-chemical characteristics of soil around industrial and agricultural area of yerraguntla, kadapa district, AP, India. International Journal Geology Earth and Environmental Sciences 3, 28-34.

Tang, J., Sun, B., Cheng, R., Shi, Z., Luo D., Liu, S., Centritto, 2019. Effects of soil nitrogen $(N)$ deficiency on photosynthetic $\mathrm{N}$-use efficiency in $\mathrm{N}$-fixing and non-
$\mathrm{N}$-fixing tree seedlings in subtropical China. Scientific Reports 9, 4604 https://doi.org/10.1038/s41598-01941035-1

Thakre, Y.G., Choudhary, M.D., Raut, R.D., 2012. Physicochemical Characterization of Red and Black Soils of Wardha Region. International Journal of Chemical and Physical Sciences 1, 60-66.

Valente, D.S.M., Queiroz, D.M., Pinto, F., Santos, N.T., Santos, F.L., 2012. Definition of Management Zones in Coffee Production Fields Based on Apparent Soil Electrical Conductivity. Scientia Agricola 69, 173-179.

Walkley, A.J., Black, I.A., 1934. Estimation of soil organic carbon by the chromic acid titration method. Soil Science 37, 29-38.

Wang, D., Mathew, W.M., Jindong, S., Xiaohui, F., Fernando, M., Dokyoung, L., Michael C., 2012. Impact of nitrogen allocation on growth and photosynthesis of Miscanthus (Miscanthus×giganteus) GCB Bioenergy 4, 688-697.

Yosuf, M., Li J., Lu J., Ren, T., Cong R., Fahad, S., Li, X., 2017. Effects of fertilization on crop production and nutrientsupplying capacity under rice-oilseed rape rotation system. Scientific reports 7, 1-9. 